\title{
Effectiveness of long-term using statins in COPD - a network meta-analysis
}

Yongbin Lu', Ruixia Chang ${ }^{2^{*}}$, Jia Yao ${ }^{1}$, Xinni X ${ }^{1}$, Yongjun Teng ${ }^{1}$ and Ning Cheng ${ }^{3^{*}}$

\begin{abstract}
Objectives: To evaluate the effectiveness of long-term treatment of statins for chronic obstructive pulmonary disease (COPD), and to answer which one is better.

Methods: General meta-analysis was performed to produce polled estimates of the effect of mortality, inflammatory factors, and lung function index in COPD patients by the search of PubMed, Web of Science, Embase, and China National Knowledge Infrastructure for eligible studies. A network meta-analysis was performed to synthetically compare the effectiveness of using different statins in COPD patients.

Results: General meta-analysis showed that using statins reduced the risk of all-cause mortality, heart diseaserelated mortality and COPD acute exacerbation (AECOPD) in COPD patients, the RR ( $95 \% \mathrm{Cl}$ ) were $0.72(0.63,0.84), 0.72$ $(0.53,0.98)$ and $0.84(0.79,0.89)$, respectively. And using statins reduced C-reactive protein (CRP) and pulmonary hypertension (PH) in COPD patients, the SMD $(95 \% \mathrm{Cl})$ were $-0.62(-0.52,-0.72)$ and $-0.71(-0.85,-0.57)$, respectively. Network meta-analysis showed that Fluvastatin (97.7\%), Atorvastatin (68.0\%) and Rosuvastatin (49.3\%) had higher cumulative probability than other statins in reducing CRP in COPD patients. Fluvastatin (76.0\%) and Atorvastatin (75.4\%) had higher cumulative probability than other satins in reducing PH in COPD patients.
\end{abstract}

Conclusions: Using statins can reduce the risk of mortality, the level of CRP and PH in COPD patients. In addition, Fluvastatin and Atorvastatin are more effective in reducing CRP and PH in COPD patients.

Keywords: Statins, Mortality, CRP, PH, Network meta-analysis

\section{Backgrounds}

Chronic obstructive pulmonary disease (COPD) affects 380 million people worldwide, representing $12 \%$ of adults over 30 years of age, the prevalence of this disease is rapidly increasing, and it will be the third cause of mortality worldwide in 2020 [1, 2]. Comorbidities associated with COPD are important aspect of the disease; in particular, cardiovascular disease (CVD) has been shown to be greater than 2 times more prevalent in COPD patients compared with normal population [3, 4]. What's more, pulmonary hypertension $(\mathrm{PH})$ is one of the most important functional derangements in COPD and leads poor prognosis, which mechanisms have been ascribed to chronic hypoxia and loss of vascular bed from

\footnotetext{
*Correspondence: 1646028404@163.com; chengn@|zu.edu.cn

²anzhou Maternal and Child Health Care Hospital, Lanzhou, Gansu 730000,

People's Republic of China

${ }^{3}$ Lanzhou University, Basic Medical College, Lanzhou, Gansu 730000, People's

Republic of China

Full list of author information is available at the end of the article
}

emphysematous destruction $[5,6]$. The importance of CVD and other comorbidities in COPD patients is such that all-cause mortality has largely become the most relevant metric for outcomes in COPD patients [7]. Localized chronic inflammation of the airways has long been observed in COPD patients, and there is a growing understanding of systemic inflammation in subset of COPD patients $[8,9]$. Specifically, high levels of CRP and IL-6 have been associated with poor outcomes in COPD patients $[10,11]$.

Statins are widely used for treatment of hypercholesterolemia and CVD, as a class of cholesterol lowering drugs. In addition to their well-established benefits for CVD, they have also been studied in a variety of other disease states, including dementia, contrast-induced nephropathy, and erectile dysfunction and COPD in most notably over the past decade $[12,13]$. What needs to be emphasized is that anti-inflammatory actions modulate the immune system and reduce inflammatory markers

(C) The Author(s). 2019 Open Access This article is distributed under the terms of the Creative Commons Attribution 4.0 International License (http://creativecommons.org/licenses/by/4.0/), which permits unrestricted use, distribution, and 
such as CRP and IL-6 are slowly being discovered [11, 14]. Systematic reviews prior to 2017 have been suggested that statins are associated with a beneficial role in treatment of COPD [15-17], which including reduce all-cause mortality, cause-specific mortality and $\mathrm{PH}$. Li et al. research which including twenty studies with a total of 303,981 patients found [16] statins reduced all-cause mortality and COPD exacerbation, but not to hospitalization. Zhang et al. which including 6 RCTS with both COPD and $\mathrm{PH}$ patients found statin therapy was associated with increased 6 -min walk test (6 $\mathrm{MW}$ ) and reduced $\mathrm{PH}$, but there was no observed difference in all-cause mortality [17]. Whether statins can reduce inflammation and $\mathrm{PH}$ or not remains controversial $[17,18]$.

Previous meta-analysis of statin involved more all-cause mortality and fewer inflammatory factors. Even if involved, it is a general proof that statins can reduce certain inflammatory mediators. Whether statins reduce inflammatory mediators and pulmonary hypertension in COPD patients, ultimately reducing patient mortality? Which statin is more effective in treating COPD patients? This network meta-analysis study the role of statins in the treatment of COPD which including short-term inflammatory mediators and long-term final outcome measures. Our network meta-analysis answers whether statins can reduce inflammation and $\mathrm{PH}$, and which statin is more effective for COPD in the first time, and provide evidence for the clinical treatment of COPD.

\section{Materials and methods}

\section{Search strategy}

We searched PubMed, Web of Science, Embase, and China National Knowledge Infrastructure for eligible studies from January 1990 to March 2018, including studies ahead of publications and without language restriction. We also performed a manual search of references cited and related in the important studies. The combinations of keywords used were ("Pulmonary Disease, Chronic Obstructive" or "Chronic Obstructive Pulmonary Disease" or "COPD" or "Chronic Obstructive Airway Disease" or "COAD" or "Chronic Obstructive Lung Disease" or "Chronic Airflow Obstruction" or "Lung Diseases, Obstructive" or "Obstructive Lung Disease" or "Obstructive Pulmonary Disease" or "Lung Disease" or "Pulmonary Disease") and ("Hydroxymethylglutaryl-CoA Reductase Inhibitors" or "HMG-CoA Reductase Inhibitors" or "Statin" or "Lovastatin" or "Pravastatin" or "Mevastatin" or "Fluvastatin" or "Atorvastatin" or "Lipitor" or "Rosuvastatin" or "pitavastatin" or "Simvastatin" or "Cerivastatin").

\section{Study selection and quality evaluation}

Studies were included in the meta-analysis if they met the following criteria: (1) Published cohort studies or randomized controlled trials (RCT); (2) Studies with $\geq 1$ months of follow-up: (3) relative risk (RR), HR estimated with $95 \% \mathrm{CI}$ or sufficient data to calculate were reported for binary variables; standardized mean difference (SMD) estimated with $95 \% \mathrm{CI}$ or sufficient data to calculate were reported for continuous variables; (4) studies in which the number of events and total number of participants in each study group were reported; (5) the outcome of interest were long-term outcome indicators including all-cause and cause-specific mortality, and short-term outcome indicators including inflammatory factors of CRP, IL-6, Interleukin-8 (IL-8) and tumor necrosis factor- $\alpha$ (TNF- $\alpha$ ), lung function index of forced expiratory volume in one second percent (FEV1\%), forced expiratory volume in one second/forced vital capacity (FEV1/FVC\%), $6 \mathrm{MW}$ and PH (measured by color Doppler echocardiography to determine tricuspid regurgitation velocity, calculated according to Bernoulli's formula $(P=4 \mathrm{~V} 2))$, blood lipids index of total cholesterol (TC) and triglyceride (TG); (6) Interventions must include two groups of statin and placebo. We excluded studies published as abstracts and review as we considered the information to be insufficient for our assessment.

Two authors independently extracted relevant information from different studies. The following data were extracted from each study: the first author's name, publish year, research methods, area, treatment, participants age, follow-up duration, the number of events and total number of participants in the intervention and control groups and outcomes as far as reported. Disagreements in data extracted were resolved by consult.

We used the Newcastle-Ottawa quality assessment scale for quality assessment of cohort studies [19]. The scale is judged based on selection (four items), comparability (one item), and exposure/outcome (three items). We used the Cochrane Collaboration risk of bias tool assessment scale for quality assessment of RCT, which including appropriateness of allocation, blinding, and management of incomplete outcome data; completeness of reporting of outcomes and other bias [20].

\section{Sensitivity analysis and publication bias}

Sensitivity analysis was performed to evaluate the Stability and reliability of the results, in which subgroup analysis was performed by statins. We compared the pooled RR and SMD estimates from different subgroups and the overall estimate. Publication bias was evaluated by Egger's test. Forest plots and funnel plots were used to examine the overall effect and assess the publication bias, respectively.

\section{Statistical methods}

Pairwise comparisons of each statin and effect versus control were performed by using random effect model 
(Stata 12.0). The random effects model is more powerful than the fixed effect model and incorporates into the weighing scheme both within-study and between-study variations [21]. We performed further subgroup analysis for inflammatory factors, lung function index and blood lipids index in the intervention and control groups. Statistical heterogeneity were evaluated by using Cochran $\mathrm{Q}$ statistic and quantified by $I^{2}$ statistic [22]. When heterogeneity was present, sensitivity analysis and subgroup analysis were performed to identify responsible outlier studies.

We used the multiple treatment meta-analysis method (MTM) proposed by Salanti et al. (a Bayesian method based on the Markov Chain Monte Carlosimulation) to compare testing modalities for effect from statins [23]. All MTMs were performed using WinBugs version 1.4.3 utilizing random effects models. The estimates obtained by generating five chains with 1000 initial iterations (burn in) and 10,000 iterations were used for estimations. We assessed the probability that which statin is best by calculating its treatment effect compared with control and counting the proportion of iterations of the Markov chain in which each statin has the highest treatment effect, second highest, and so on. We developed rankograms and cumulative probability plots to graphically present the distribution of ranking probabilities and estimated the surface under the cumulative ranking (SUCRA) line for each statin [24].

\section{Results}

\section{Characteristics of eligible studies}

A total of 988 studies were identified in the original database search, of which 138 needed to be further screened. Finally, 53 studies were included (Additional file 1: Figure $\mathrm{S} 1$ ).

Of the 53 studies, 14 studies [11, 25-37] reported all-cause mortality, 4 studies [26, 30, 36, 38] reported heart disease-related mortality, 6 studies [27, 30, 34, 35, 38, 39] reported COPD mortality, 12 studies [11, 25, 28, 30, 38, 40-46] reported AECOPD, 20 studies [44, 4664] reported CRP, 12 studies [44, 47, 50, 51, 54, 59, 62, 65-69] reported IL-6, 5 studies [55, 59, 60, 65, 70] reported IL-8, 9 studies [51, 53, 58-62, 65, 68] reported TNF- $\alpha, 17$ studies $[5,45,47,49-52,57,63,65,67$, 68, 71-75] reported FEV1\%, 15 studies [5, 50, 51, 55, $59,60,65,67,68,70-75]$ reported FEV1/FVC\%, 7 studies $[5,52,60-62,66,70]$ reported $6 \mathrm{MW}, 9$ studies $[36,47,48,51,58,66,70,71,74]$ reported TC, 6 studies $[47,48,66,70,71,74]$ reported TG and 10 studies $[5,45,52,55,56,60,63,66,73,74]$ reported $\mathrm{PH}$. The number of patients in each studies ranged from 40 to 68,754 . The duration of follow-up ranged from one to 120 months (Table 1).

\section{Using statins reduced the risk of all-cause mortality and} cause-specific mortality

Random effect models analyses showed that using statins significantly reduced the risk of all-cause mortality, the RR (95\% CI) was $0.72(0.63,0.84)$, with a significant strong heterogeneity $\left(I^{2}=86.8 \%, P<0.01\right)$. Figure 1 showed the heterogeneity reduced when using sensitivity analysis and exclude a study [30] $\left(I^{2}=67.2 \%, P<0.01\right)$, the RR $(95 \% \mathrm{CI})$ was $0.71(0.62,0.80)$. Using statins didn't reduced the risk of heart disease-related mortality, the RR $(95 \% \mathrm{CI})$ was $0.92(0.83,1.03)$ (Additional file 2: Figure S2). Using statins reduced the risk of COPD mortality and AECOPD, the RR $(95 \% \mathrm{CI})$ were $0.72(0.53$, $0.98)$ and $0.84(0.79,0.89)$, respectively (Additional file 3 : Figure S3 and Additional file 4: Figure S4).

\section{Using statins reduced the level of CRP, IL-6, IL-8 and TNF-a}

Figure 2 showed the changes of CRP after using statins in COPD patients, which showed statins therapy significantly reduced CRP, the SMD (95\% CI) was - 0.62 (0.52,-0.72). Subgroup analysis showed Pravastatin, Simvastatin, Atorvastatin, Rosuvastatin, Fluvastatin were significantly reduced CRP, the SMD $(95 \% \mathrm{CI})$ were -0.36 $(-0.72,-0.01),-0.54(-0.68,-0.39),-0.72(-0.89,-0.55)$, $-0.57(-0.88,-0.27)$ and $-1.66(-0.2 .21,-1.12)$, respectively. Figure 3 showed the diagram of network meta-analysis between the SMD changes of CRP after using different statins in COPD patients. Network meta-analysis showed that Fluvastatin significantly reduced CRP compared Pravastatin and Simvastatin, the SMD $(95 \% \mathrm{CI})$ were $-1.28(-2.59,-0.01)$ and $-1.10(-$ $2.07,-0.11$ ), respectively. The SUCRA of reducing the CRP in COPD patients in Atorvastatin, Fluvastatin, Rosuvastatin, Pravastatin and Simvastatin were 68.0, 97.7, 49.3, 33.9 and 46.3\%, respectively (Table 2 and Fig. 4). Across all patients, Fluvastatin, Atorvastatin and Rosuvastatin had higher overall probability, and they were more effective in reducing CRP than other statins in COPD patients.

Additional file 5: Figure S5 showed that statins therapy significantly reduced IL-6, the SMD (95\% CI) was -0.95 $(-1.09,-0.81)$. Subgroup analysis showed Pravastatin (1 study), Rosuvastatin, Simvastatin and Atorvastatin were significantly reduced IL-6, and the SMD (95\% CI) were $-1.00 \quad(-1.38,-0.63), \quad-1.34 \quad(-1.79,-0.89),-1.25 \quad$ ($1.56,-0.95)$ and $-0.79(-1.01,-0.58)$, respectively. Additional file 6: Figure S6 showed the diagram of network meta-analysis between the SMD changes of IL-6 after using different statins in COPD patients. Network meta-analysis showed that the SUCRA of reducing the IL-6 in COPD patients in Atorvastatin, Fluvastatin, Rosuvastatin, Pravastatin and Simvastatin were 52.7, 25.5, 70.7, 58.3 and 79.9\%, respectively (Additional file 7: 
Table 1 Study characteristic

\begin{tabular}{|c|c|c|c|c|c|c|c|c|c|c|c|}
\hline Author & $\begin{array}{l}\text { Publish } \\
\text { year }\end{array}$ & $\begin{array}{l}\text { Research } \\
\text { methods }\end{array}$ & Area & Disease & Treatment & Number & Age(years) & $\begin{array}{l}\text { Treatment } \\
\text { times(months) }\end{array}$ & Dose & $\begin{array}{l}\text { Number } \\
\text { of } \\
\text { smokers }\end{array}$ & Outcomes \\
\hline Chin-Chou Huang & 2011 & Cohort & Taiwan & COPD & Statin/Conventional treatment & $6252 / 12469$ & $64 / 64$ & 55 & NA & NA & $\mathrm{d}$ \\
\hline Konstantinos Bartziokas & 2011 & Cohort & Greece & COPD & Statin/Conventional treatment & $74 / 171$ & $71.4 \pm 9.1 / 71.1 \pm 9.8$ & 12 & NA & $29 / 64$ & $\mathrm{a}, \mathrm{d}$ \\
\hline Andrea Rossi & 2017 & Cohort & Italy & COPD & Rosuvastatin/Conventional treatment & $538 / 522$ & $71 \pm 9 / 72 \pm 9$ & 47 & $10 \mathrm{mg} /$ day & $359 / 327$ & $\mathrm{a}, \mathrm{b}$ \\
\hline Truls S Ingebrigtsen & 2014 & RCT & Denmark & COPD & Statin/Conventional treatment & $320 / 1226$ & $71 / 66$ & 36 & $\mathrm{NA}$ & $\mathrm{NA}$ & d \\
\hline Y R B M van Gestel & 2009 & RCT & Netherlands & COPD & Statin/Conventional treatment & $341 / 969$ & $66 \pm 12 / 66 \pm 12$ & 60 & $\mathrm{NA}$ & $\mathrm{NA}$ & $\mathrm{a}, \mathrm{c}$ \\
\hline Seyed Ali Javad Moosavi & 2013 & RCT & Iran & $\mathrm{COPD}+\mathrm{PH}$ & Atorvastatin/Conventional treatment & $24 / 21$ & $65 \pm 11 / 68 \pm 14$ & 6 & $40 \mathrm{mg} / \mathrm{day}$ & NA & $\mathrm{i}, \mathrm{j}, \mathrm{k}, \mathrm{l}$ \\
\hline Yvette R.B.M. van Gestel & 2008 & Cohort & United Kingdom & $\mathrm{COPD}+\mathrm{PH}$ & Statin/Conventional treatment & $330 / 980$ & $69 \pm 9 / 69 \pm 10$ & 120 & $\mathrm{NA}$ & $\mathrm{NA}$ & $\mathrm{a}, \mathrm{d}$ \\
\hline Lies Lahousse & 2012 & Cohort & Netherlands & COPD & Statin/Conventional treatment & $363 / 2345$ & $81 \pm 2.5 / 78 \pm 1.75$ & 24 & NA & $309 / 1990$ & $\mathrm{a}, \mathrm{d}$ \\
\hline Eric M Mortensen & 2009 & RCT & American & COPD & Statin/Conventional treatment & $2286 / 8926$ & $73.5 \pm 5.8 / 74.3 \pm 5.8$ & 3 & NA & NA & a \\
\hline Mayank Ajmera & 2016 & Cohort & American & COPD & Statin/Conventional treatment & $5771 / 13289$ & $40-64 / 40-64$ & 48 & NA & NA & d \\
\hline G.J. Criner & 2014 & Cohort & American + Canada & COPD & Simvastatin/Conventional treatment & $433 / 452$ & $62.2 \pm 8.5 / 62.3 \pm 8.4$ & 36 & $40 \mathrm{mg} /$ day & $133 / 143$ & $\mathrm{a}, \mathrm{b}, \mathrm{c}, \mathrm{d}$ \\
\hline Carlene MM Lawesa & 2011 & Cohort & new Zealand & COPD & Statin/Conventional treatment & $596 / 1091$ & $50-80 / 50-80$ & 48 & NA & NA & a \\
\hline Meng-Ting Wang & 2013 & Cohort & China & COPD & Statin/Conventional treatment & $1584 / 5950$ & $74.6 \pm 9.6 / 74.1 \pm 9.4$ & 30 & NA & NA & d \\
\hline Magnus P & 2012 & Cohort & Sweden & COPD & Statin/Conventional treatment & $431 / 965$ & $73.5 \pm 7.1 / 74.1 \pm 8.6$ & 48 & $\mathrm{NA}$ & $\mathrm{NA}$ & a \\
\hline V. Søyseth & 2007 & Cohort & Norway & COPD & Statin/Conventional treatment & $118 / 736$ & $68.5 \pm 8.4 / 71.1 \pm 11.6$ & 23 & $\mathrm{NA}$ & $\mathrm{NA}$ & a \\
\hline A.J.N. Raymakers & 2017 & Cohort & Canada & COPD & Statin/Conventional treatment & $7566 / 32112$ & $69.9 \pm 8.8 / 70.5 \pm 11.4$ & 18 & NA & NA & $\mathrm{a}, \mathrm{c}$ \\
\hline Floyd J. Frost & 2007 & Cohort & American & COPD & Statin/Conventional treatment & $11583 / 57174$ & $\mathrm{NA} / \mathrm{NA}$ & 12 & $>4 \mathrm{mg} /$ day & $\mathrm{NA}$ & $\mathrm{a}, \mathrm{c}$ \\
\hline Xia Sheng & 2012 & Cohort & United Kingdom & COPD & Statin/Conventional treatment & $617 / 657$ & $68.5 \pm 8.8 / 68.7 \pm 11.8$ & 12 & NA & NA & $\mathrm{a}, \mathrm{b}, \mathrm{m}$ \\
\hline Ju-Chi Liu & 2016 & Cohort & China & COPD & Statin/Conventional treatment & $10086 / 33716$ & $61.55 \pm 10.97 / 63.33 \pm 13.74$ & 12 & $\mathrm{NA}$ & NA & $\mathrm{c}$ \\
\hline Fengxia Yu & 2012 & RCT & China & $\mathrm{COPD}+\mathrm{PH}$ & Atorvastatin/Conventional treatment & $78 / 78$ & $65.15 \pm 6.60 / 64.80 \pm 6.70$ & 12 & $10 \mathrm{mg} / \mathrm{d}$ & NA & $\mathrm{e}, \mathrm{i}, \mathrm{k}, 1$ \\
\hline Longjun Mao & 2017 & RCT & China & $\mathrm{COPD}+\mathrm{PH}$ & Atorvastatin/Conventional treatment & $53 / 53$ & $51.28 \pm 3.12 / 52.42 \pm 3.29$ & 3 & $20 \mathrm{mg} / \mathrm{d}$ & NA & $\mathrm{d}, \mathrm{e}, \mathrm{f}$ \\
\hline Hang Ma & 2014 & RCT & China & $\mathrm{COPD}+\mathrm{PH}$ & $\begin{array}{l}\text { Atorvastatin/Conventional treatment } \\
\text { Atorvastatin/Simvastatin/ }\end{array}$ & $23 / 22$ & $69.87 \pm 6.87 / 69.87 \pm 6.87$ & 6 & $20 \mathrm{mg} / \mathrm{d}$ & NA & $\mathrm{f}, \mathrm{k}, \mathrm{l}, \mathrm{m}, \mathrm{n}$ \\
\hline Dong Pang & 2015 & RCT & China & COPD & Conventional treatment & $40 / 40 / 40$ & $44-78 / 45-77 / 43-79$ & 6 & $20 / 40 \mathrm{mg} / \mathrm{d}$ & NA & $\mathrm{b}, \mathrm{c}, \mathrm{d}$ \\
\hline Dan Cheng & 2016 & RCT & China & $\mathrm{COPD}+\mathrm{PH}$ & Atorvastatin/Conventional treatment & $35 / 41$ & $66 \pm 4.0 / 67 \pm 4.8$ & 6 & $20 \mathrm{mg} / \mathrm{d}$ & $\mathrm{NA}$ & $\mathrm{i}, \mathrm{j}, 1$ \\
\hline Lina Niu & 2015 & RCT & China & $\mathrm{COPD}+\mathrm{PH}$ & Atorvastatin/Conventional treatment & $50 / 50$ & $45.22 \pm 14.29 / 45.34 \pm 14.46$ & 6 & $20 \mathrm{mg} / \mathrm{d}$ & NA & $\mathrm{e}, \mathrm{g}, \mathrm{j}, \mathrm{l}$ \\
\hline Lingling Wang & 2010 & RCT & China & $\mathrm{COPD}+\mathrm{PH}$ & Fluvastatin/Conventional treatment & $35 / 35$ & $64 \pm 3.5 / 63 \pm 5.2$ & 6 & $40 \mathrm{mg} / \mathrm{d}$ & NA & $\mathrm{e}, 1$ \\
\hline Wei Wang & 2012 & RCT & China & $\mathrm{COPD}+\mathrm{PH}$ & Fluvastatin/Conventional treatment & $56 / 56$ & $62.4 \pm 7.3 / 68.4 \pm 8.5$ & 6 & $40 \mathrm{mg} / \mathrm{d}$ & $9 / 13$ & $\mathrm{i}, \mathrm{j}, \mathrm{l}, \mathrm{m}, \mathrm{n}$ \\
\hline Yan Ding & 2016 & RCT & China & $\mathrm{COPD}+\mathrm{PH}$ & Simvastatin/Conventional treatment & $50 / 50$ & $70.29 \pm 7.86 / 70.15 \pm 7.62$ & 6 & $20 \mathrm{mg} / \mathrm{d}$ & $\mathrm{NA}$ & $\mathrm{d}, \mathrm{i}, 1$ \\
\hline Chen Tang & 2017 & RCT & China & $\mathrm{COPD}+\mathrm{PH}$ & Simvastatin/Conventional treatment & $43 / 43$ & $61.7 \pm 9.4 / 60.8 \pm 8.9$ & 6 & $20 \mathrm{mg} / \mathrm{d}$ & $\mathrm{NA}$ & $\mathrm{e}, \mathrm{g}, \mathrm{h}, \mathrm{j}, \mathrm{k}, \mathrm{l}$ \\
\hline Yiyun Wu & 2016 & RCT & China & COPD & Simvastatin/Conventional treatment & $50 / 50$ & $47.4 \pm 5.2 / 48.4 \pm 5.4$ & 12 & $20 \mathrm{mg} / \mathrm{d}$ & $\mathrm{NA}$ & $\mathrm{d}, \mathrm{e}$ \\
\hline Juan Sun & 2014 & RCT & China & $\mathrm{COPD}+\mathrm{PH}$ & Simvastatin/Conventional treatment & $40 / 40$ & $55-86 / 56-88$ & 3 & $20 \mathrm{mg} / \mathrm{d}$ & NA & $\mathrm{e}, \mathrm{i}, 1$ \\
\hline Tsung-Ming Lee & 2008 & RCT & American & COPD & Pravastatin/Conventional treatment & $62 / 63$ & $70 \pm 8 / 71 \pm 6$ & 6 & $40 \mathrm{mg} /$ day & $50 / 48$ & $\mathrm{e}, \mathrm{f}, \mathrm{i}, \mathrm{m}, \mathrm{n}$ \\
\hline Anetta Undas & 2009 & RCT & Poland & COPD & Simvastatin/Conventional treatment & $26 / 23$ & $64.9 \pm 9.2 / 64.9 \pm 9.2$ & 3 & $40 \mathrm{mg} /$ day & $10 / 10$ & $\mathrm{e}, \mathrm{m}, \mathrm{n}$ \\
\hline Hassan Ghobadi & 2014 & RCT & Iran & COPD & Atorvastatin/Conventional treatment & $23 / 22$ & $47.3 \pm 7.5 / 50.2 \pm 8.2$ & 3 & $40 \mathrm{mg} / \mathrm{day}$ & NA & $\mathrm{e}, \mathrm{i}$ \\
\hline A. Neukamm & 2015 & RCT & Norway & COPD & Rosuvastatin/Conventional treatment & $47 / 47$ & $66 \pm 0.8 / 63 \pm 1.0$ & 3 & $10 \mathrm{mg} /$ day & NA & $\mathrm{e}, \mathrm{f}, \mathrm{i}, \mathrm{j}$ \\
\hline Przemysław Kaczmarek & 2010 & RCT & Poland & COPD & Simvastatin/Conventional treatment & $28 / 28$ & $66.10 \pm 11.42 / 63.82 \pm 8.37$ & 3 & $40 \mathrm{mg} / \mathrm{day}$ & NA & $\mathrm{e}, \mathrm{f}, \mathrm{h}, \mathrm{i}, \mathrm{j}, \mathrm{m}$ \\
\hline Wei Zheng & 2013 & RCT & China & COPD & Atorvastatin/Conventional treatment & $43 / 44$ & $63.8 \pm 7.6 / 63.8 \pm 7.6$ & 6 & $20 \mathrm{mg} / \mathrm{d}$ & NA & $f, g, h, i, j$ \\
\hline Sujuan Guo & 2015 & RCT & China & COPD & Atorvastatin/Conventional treatment & $50 / 50$ & $56.27 \pm 10.33 / 57.42 \pm 10.19$ & 3 & $20 \mathrm{mg} / \mathrm{d}$ & NA & $\mathrm{e}, \mathrm{h}$ \\
\hline Rongchang Zhang & 2015 & RCT & China & COPD & Atorvastatin/Conventional treatment & $30 / 30$ & $60.5 \pm 8.9 / 61.2 \pm 7.8$ & 12 & $20 \mathrm{mg} / \mathrm{d}$ & NA & $\mathrm{e}, \mathrm{f}$ \\
\hline Jianrong $\mathrm{Xu}$ & 2012 & RCT & China & COPD & Fluvastatin/Conventional treatment & $20 / 20$ & $64.5 \pm 7.5 / 64.5 \pm 7.5$ & 6 & $20 \mathrm{mg} / \mathrm{d}$ & NA & $\mathrm{f}, \mathrm{i}, \mathrm{j}$ \\
\hline Zhenghua Gong & 2015 & RCT & China & COPD & Rosuvastatin/Conventional treatment & $40 / 42$ & $72.3 \pm 12.8 / 71.5 \pm 13.4$ & 4 & $20 \mathrm{mg} / \mathrm{d}$ & NA & $\mathrm{g}, \mathrm{j}, \mathrm{k}, \mathrm{m}, \mathrm{n}$ \\
\hline Wei Dong & 2015 & RCT & China & COPD & Rosuvastatin/Conventional treatment & $41 / 42$ & $60.43 \pm 2.35 / 61.14 \pm 5.63$ & 12 & $10 \mathrm{mg} / \mathrm{d}$ & NA & $\mathrm{e}, \mathrm{i}$ \\
\hline Xuelin Men & 2011 & RCT & China & COPD & Simvastatin/Conventional treatment & $41 / 45$ & $72.0 \pm 7.6 / 70.0 \pm 9.8$ & 3 & $20 \mathrm{mg} / \mathrm{d}$ & NA & $\mathrm{e}, \mathrm{h}, \mathrm{m}$ \\
\hline Zhimin Jiang & 2013 & RCT & China & COPD & Simvastatin/Conventional treatment & $20 / 20$ & $52-78 / 52-78$ & 6 & $20 \mathrm{mg} / \mathrm{d}$ & NA & $\mathrm{f}, \mathrm{h}, \mathrm{i}, \mathrm{j}$ \\
\hline Wei Zeng & 2016 & RCT & China & COPD & Simvastatin/Conventional treatment & $40 / 40$ & $65.4 \pm 7.8 / 64.9 \pm 7.6$ & 6 & $20 \mathrm{mg} / \mathrm{d}$ & $\mathrm{NA}$ & $\mathrm{e}, \mathrm{f}, \mathrm{g}, \mathrm{h}, \mathrm{j}$ \\
\hline Ruihua Zhang & 2014 & RCT & China & $\mathrm{COPD}+\mathrm{PH}$ & Simvastatin/Conventional treatment & $45 / 45$ & $69.88 \pm 6.84 / 65.48 \pm 6.13$ & 6 & $20 \mathrm{mg} / \mathrm{d}$ & NA & $\mathrm{e}, \mathrm{h}, \mathrm{k}$ \\
\hline Hong Lv & 2017 & RCT & China & COPD & Simvastatin/Conventional treatment & $30 / 32$ & $70.21 \pm 4.67 / 70.21 \pm 4.67$ & 3 & $20 \mathrm{mg} / \mathrm{d}$ & NA & $\mathrm{e}, \mathrm{f}, \mathrm{h}, \mathrm{k}$ \\
\hline Tsung-Ming LEE & 2009 & RCT & Taiwan & $\mathrm{COPD}+\mathrm{PH}$ & Pravastatin/Conventional treatment & $27 / 26$ & $71 \pm 8 / 72 \pm 6$ & 6 & $40 \mathrm{mg} / \mathrm{day}$ & $22 / 21$ & $\mathrm{I}, \mathrm{j}, \mathrm{m}, \mathrm{n}$ \\
\hline Qing Ye & 2015 & RCT & China & $\mathrm{COPD}+\mathrm{PH}$ & Simvastatin/Conventional treatment & $30 / 30$ & $58.9 \pm 7.5 / 59.4 \pm 6.8$ & 1 & $20 \mathrm{mg} / \mathrm{d}$ & $\mathrm{NA}$ & $\mathrm{e}$ \\
\hline Tse-Hsuan Su & 2017 & Cohort & Taiwan & COPD & statin/Conventional treatment & $1921 / 4849$ & $77 \pm 6.5 / 67 \pm 5.9$ & 120 & NA & NA & a \\
\hline Shanshan Cheng & 2015 & RCT & China & COPD & Atorvastatin/Conventional treatment & $56 / 56$ & $64.17 \pm 8.14 / 61.70 \pm 8.79$ & 2 & $20 \mathrm{mg} / \mathrm{d}$ & $18 / 22$ & $i, j$ \\
\hline Yong Ying & 2013 & RCT & China & COPD & Atorvastatin/Conventional treatment & $40 / 40$ & $68.7 \pm 6.1 / 69.1 \pm 5.9$ & 1 & $20 \mathrm{mg} / \mathrm{d}$ & NA & $\mathrm{f}$ \\
\hline Guangyang Zheng & 2017 & RCT & China & COPD & Simvastatin/Conventional treatment & $90 / 90$ & $72.9 \pm 8.7 / 70.2 \pm 8.1$ & 8 & $20 \mathrm{mg} / \mathrm{d}$ & NA & $\mathrm{i}, \mathrm{j}$ \\
\hline
\end{tabular}

a:all-cause mortality;b:heart disease-related mortality;c:COPD mortality;d:COPD aggravation;e:CRP;:fIL-6;g:IL-8;h:TNF-a;:FEV1\%;j;FEV1/FVC\%;k:6WMD;l:PH;m:TC;n:TG 


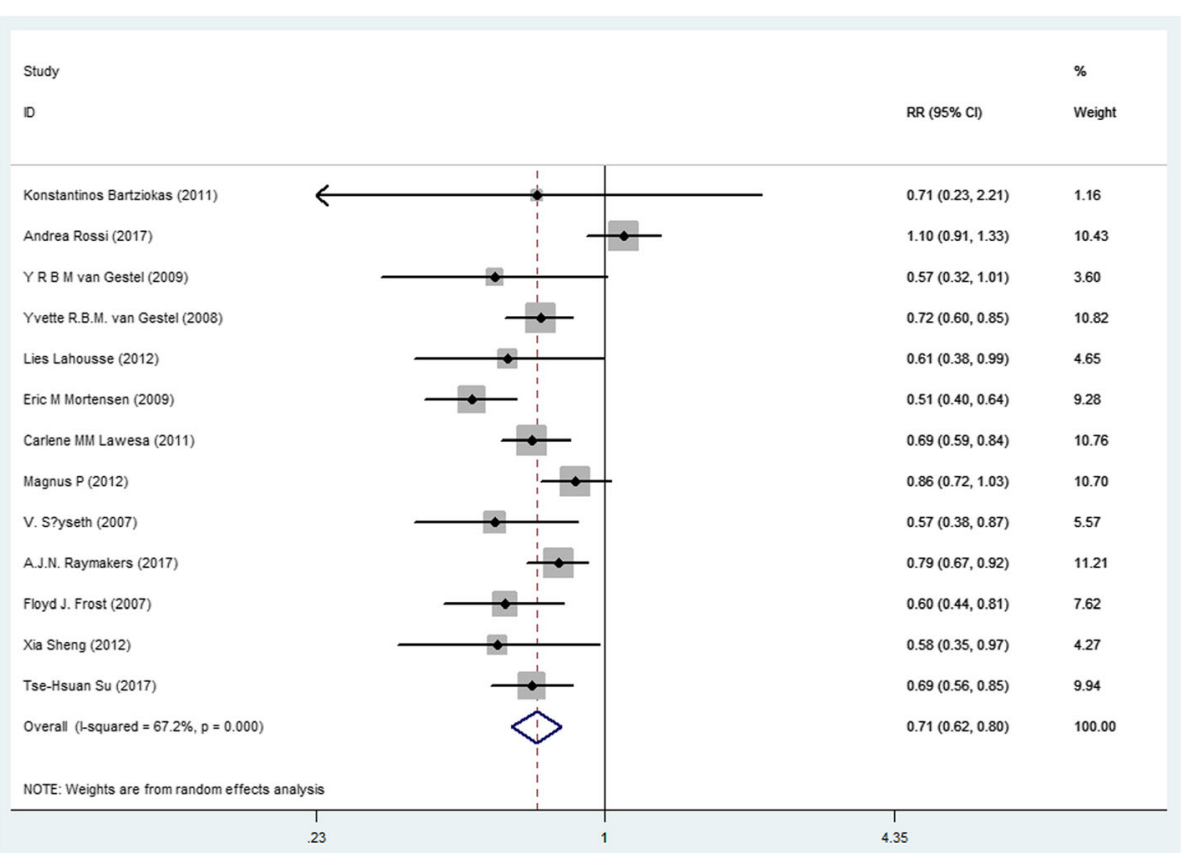

Fig. 1 Forest plot showing effect of statins on all-cause mortality in COPD patients

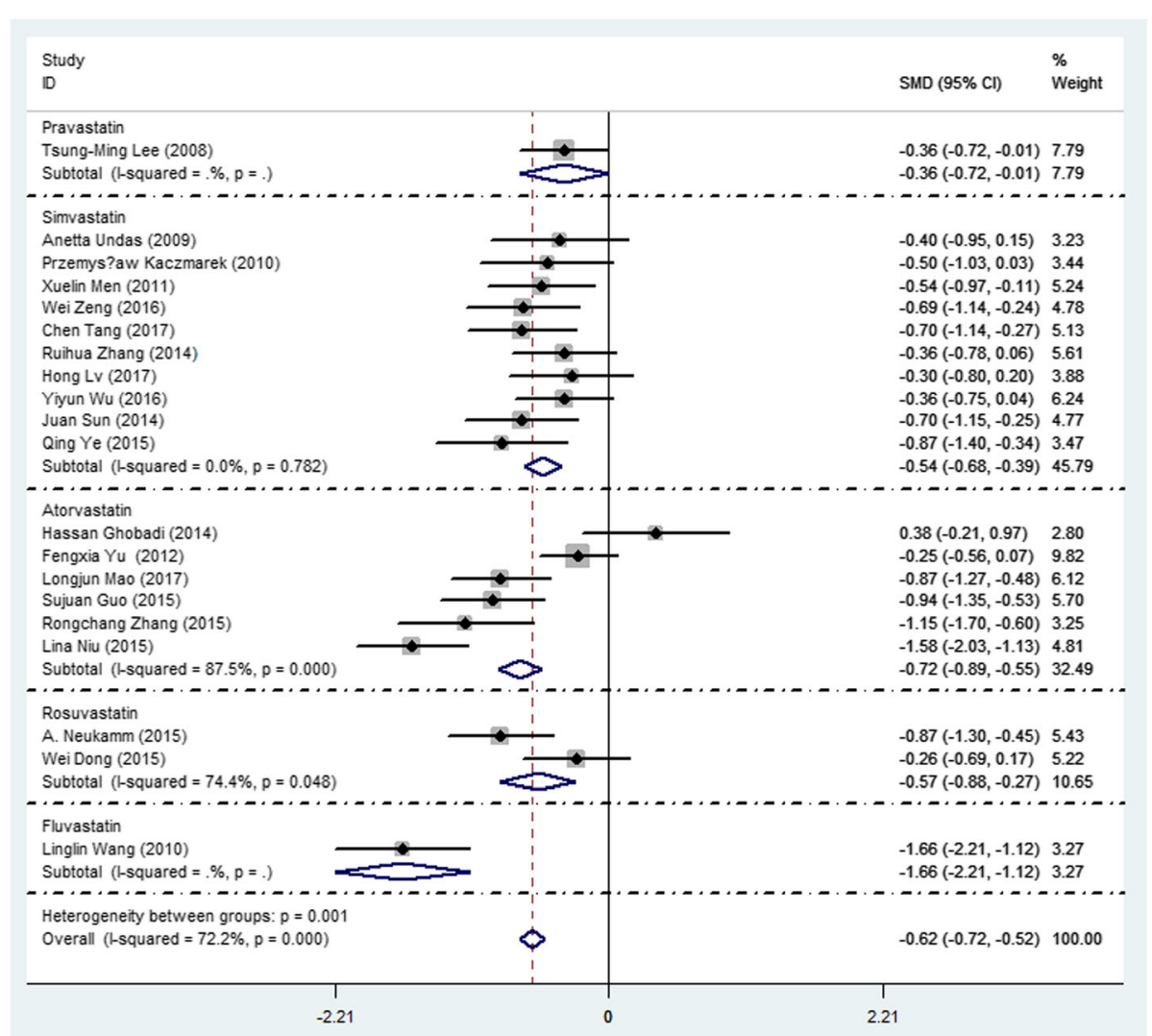

Fig. 2 Forest plot showing effect of statins on CRP in COPD patients 


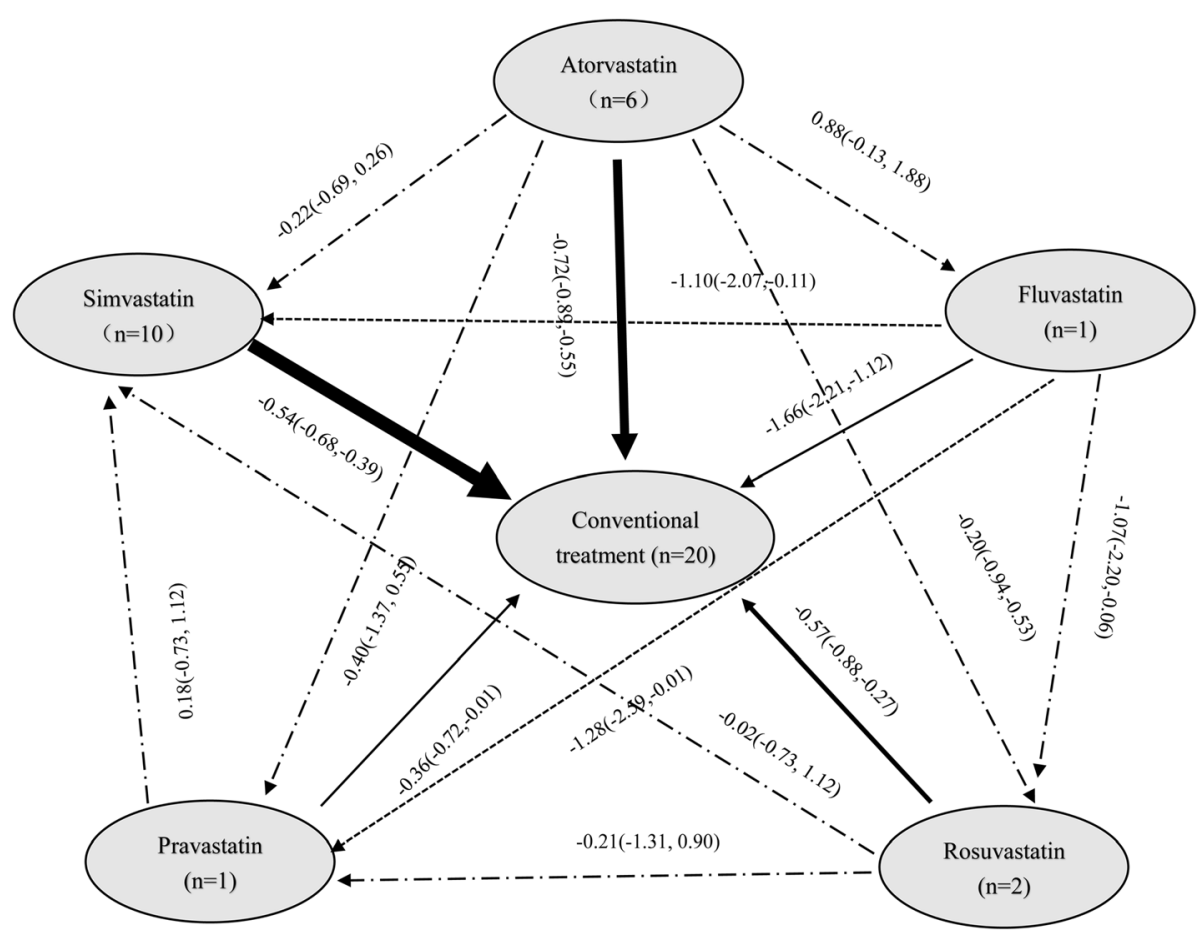

Fig. 3 Evidence network of the effect of using statins on CRP levels in COPD patients

Table S1 and Additional file 8: Figure S7). Across all patients, Simvastatin, Rosuvastatin and Pravastatin had higher overall probability, and they were more effective in reducing IL-6 than other statins in COPD patients.

Additional file 9: Figure S8 showed that statins therapy significantly reduced IL- 8 , the SMD $(95 \% \mathrm{CI})$ was -0.55 $(-74,-0.35)$, and with a significant moderate heterogeneity $\left(I^{2}=80.4 \%\right)$. The heterogeneity significant reduced when using sensitivity analysis and exclude one studies [59] $\left(I^{2}=0.0 \%\right)$, the SMD $(95 \% \mathrm{CI})$ was $-0.37(-0.58,-0.16)$. Subgroup analysis showed Atorvastatin, Rosuvastatin and Simvastatin were significantly reduced IL-8, and the SMD $(95 \% \mathrm{CI})$ were $-0.28(-0.57,0.01),-0.62(-1.06,-0.17)$ and $-0.86(-1.18,-0.53)$, respectively.

Additional file 10: Figure S9 showed that statins therapy significantly reduced TNF- $\alpha$, the SMD (95\% CI) was $0.68(-0.83,-0.52)$, with a significant moderate heterogeneity $\left(I^{2}=82.3 \%\right)$. The heterogeneity significant reduced

Table 2 Rank probability analysis of CRP with using stains in COPD patients

\begin{tabular}{llllll}
\hline Treatment & SUCRA & sd & $2.50 \%$ & median & $97.50 \%$ \\
\hline Atorvastatin & 68.0 & 0.1685 & 0.2 & 0.8 & 1.0 \\
Fluvastatin & 97.7 & 0.0969 & 0.6 & 1.0 & 1.0 \\
Rosuvastatin & 49.3 & 0.2392 & 0.0 & 0.4 & 0.8 \\
Pravastatin & 33.9 & 0.2718 & 0.0 & 0.2 & 0.8 \\
Simvastatin & 46.3 & 0.1759 & 0.2 & 0.4 & 0.8 \\
Conventional treatment & 4.8 & 0.0900 & 0.0 & 0.0 & 0.2 \\
\hline
\end{tabular}

when using sensitivity analysis and exclude one study [59] $\left(I^{2}=65.2 \%\right)$, the SMD $(95 \% \mathrm{CI})$ was $-0.56(-0.72,-0.39)$. Subgroup analysis showed Simvastatin and Atorvastatin were significantly reduced TNF- $\alpha$, and the SMD $(95 \% \mathrm{CI})$ were $-0.79(-0.98,-0.60)$ and $-0.40 \quad(-0.69,-0.11)$, respectively. Additional file 11: Figure S10 showed the diagram of network meta-analysis between the changes of TNF- $\alpha$ after using different statins in COPD patients. Network meta-analysis showed that the SUCRA of reducing the TNF- $\alpha$ in COPD patients in Atorvastatin and Simvastatin were 51.0 and $89.2 \%$, respectively (Additional file 12: Table S2 and Additional file 13: Figure S11). Across all patients, Simvastatin had higher overall probability, and it was more effective in reducing TNF- $\alpha$ than Atorvastatin in COPD patients.

\section{Using statins reduced the level of $\mathrm{PH}$}

Figure 5 showed that statins significantly reduced $\mathrm{PH}$, the SMD $(95 \% \mathrm{CI})$ was $-0.71(-0.85,-0.57)$, with a significant lower heterogeneity $\left(I^{2}=45.9 \%\right)$. Subgroup analysis showed Simvastatin, Atorvastatin and Fluvastatin significantly reduced $\mathrm{PH}$, and the SMD (95\% CI) were $-0.54 \quad(-0.79,-0.30),-0.79 \quad(-0.99,-0.59)$ and $0.77(-1.07,-0.46)$, respectively. Figure 6 showed the diagram of network meta-analysis between the changes of $\mathrm{PH}$ after using different statins in COPD patients. Network meta-analysis showed, the SUCRA of reducing the $\mathrm{PH}$ in COPD patients in Atorvastatin, Fluvastatin and Simvastatin were $75.4,76.0$ and $48.0 \%$, respectively 

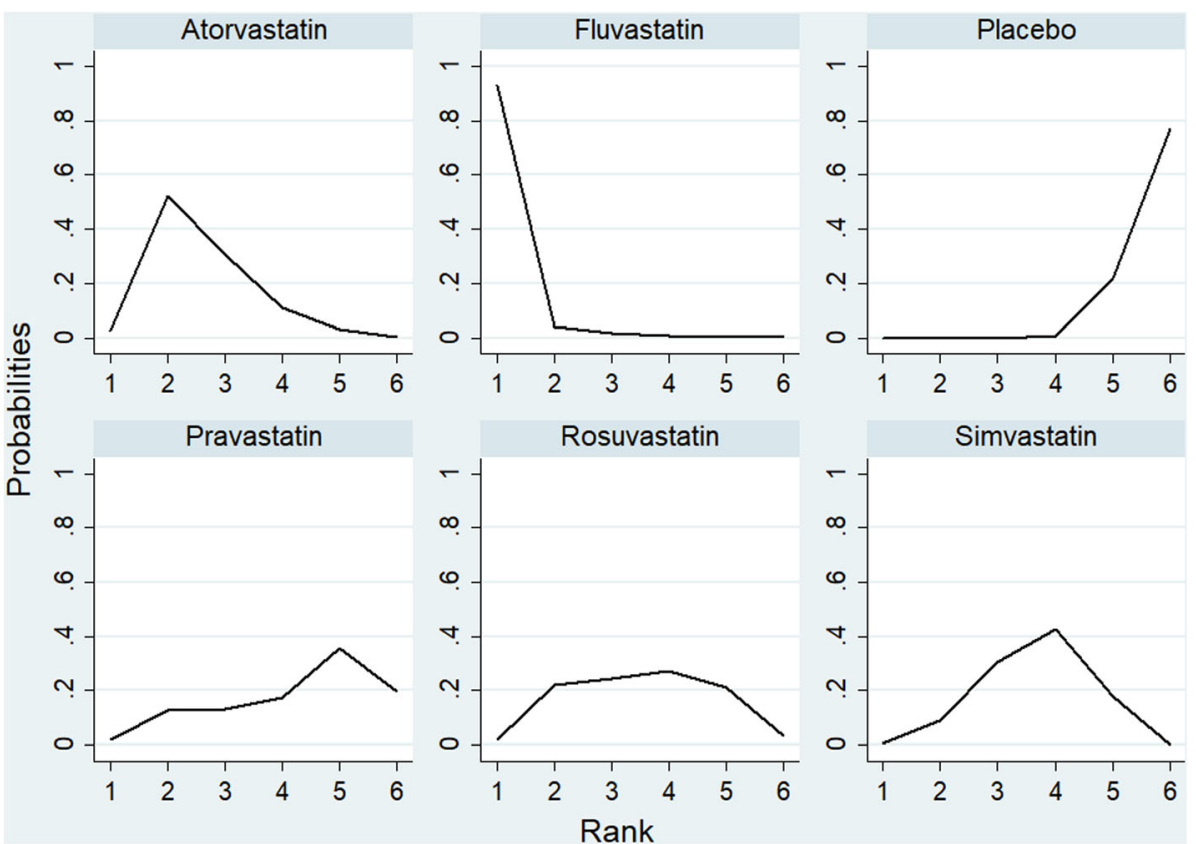

Fig. 4 Rank probability analysis of CRP with using statins in COPD patients

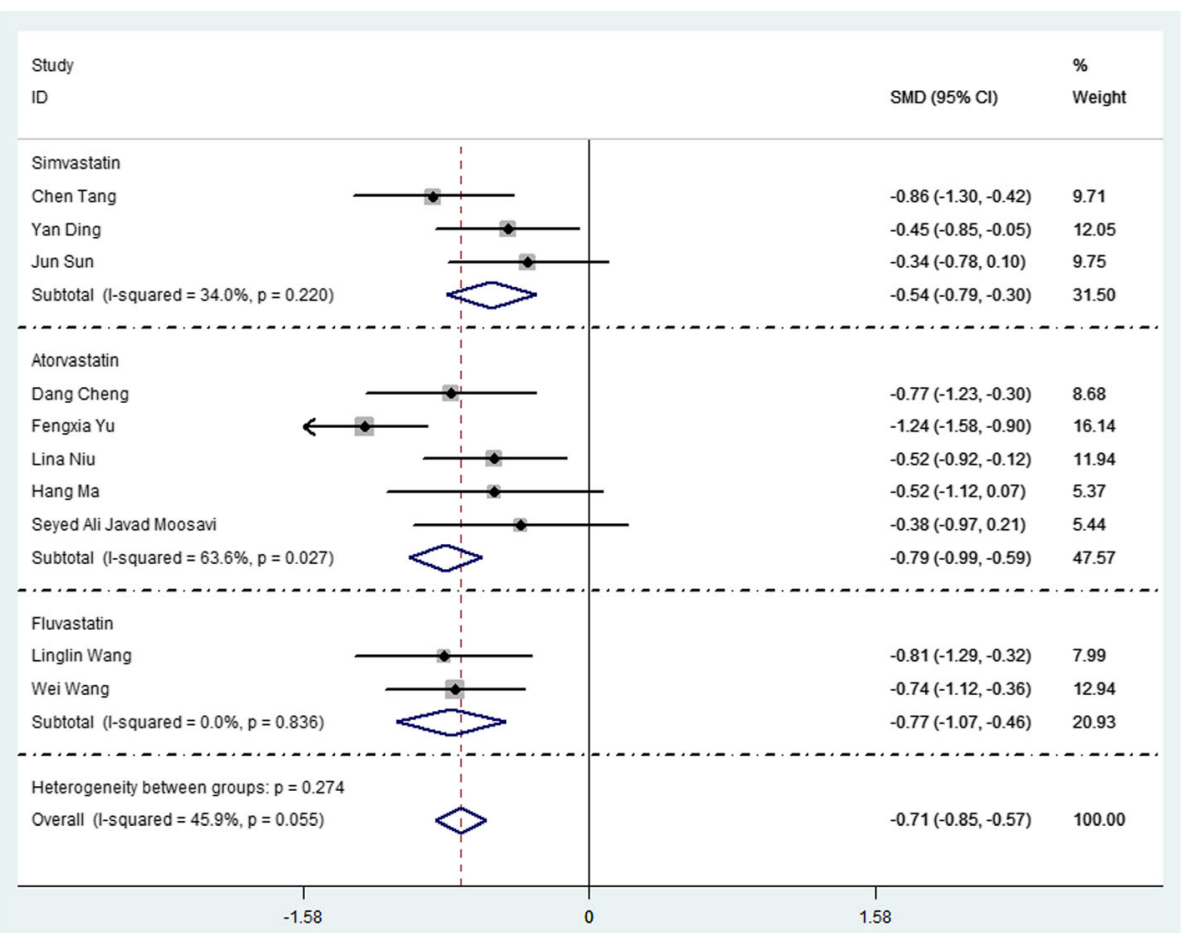

Fig. 5 Forest plot showing effect of statins on PH in COPD patients 


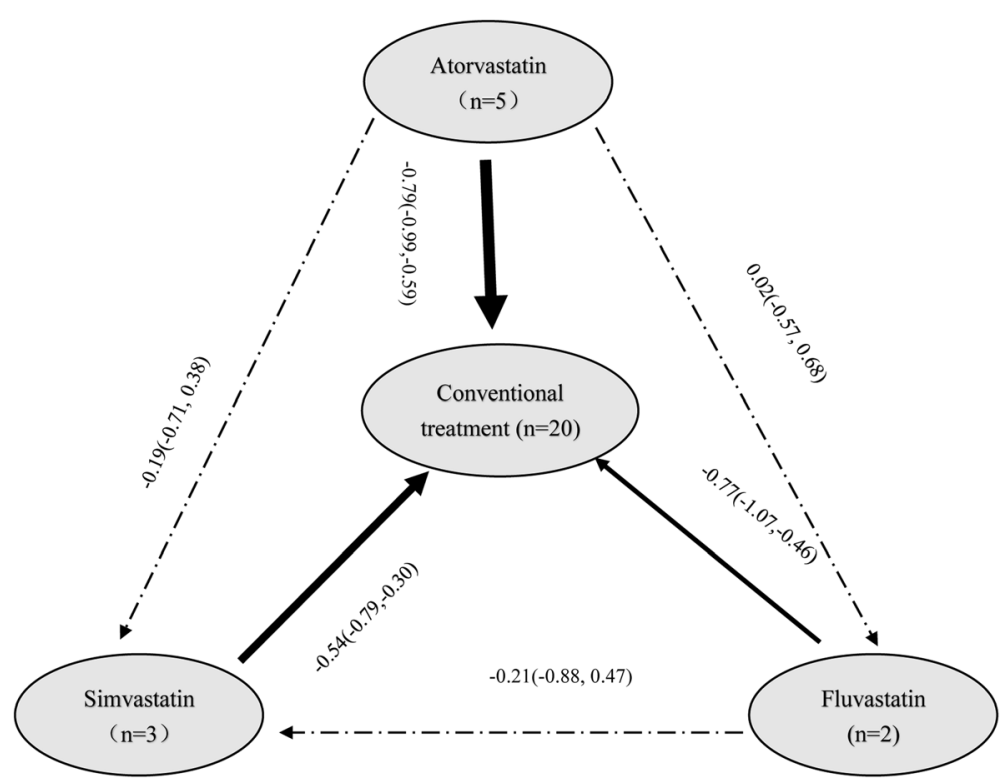

Fig. 6 Evidence network of the effect of using statins on PH levels in COPD patients

(Table 3 and Fig. 7). Across all patients, Fluvastatin and Atorvastatin had higher overall probability, and they were more effective in increasing FEV\% than Simvastatin in COPD patients.

Additional file 14: Figure S12 showed that statins significantly increased FEV1\%, the SMD (95\% CI) was 0.38 $(0.28,0.49)$, with a significant moderate heterogeneity $\left(I^{2}=66.0 \%\right)$. The heterogeneity significantly reduced when using sensitivity analysis and exclude one study [74] $\left(I^{2}=36.9 \%\right)$, the SMD (95\% CI) was $0.46(0.35$, 0.57). Subgroup analysis showed Pravastatin, Rosuvastatin, Simvastatin and Atorvastatin were significantly increased FEV1\%, and the SMD (95\% CI) were $0.29(0.09$, $0.50), \quad 0.72(0.41,1.02), 0.41(0.22,0.59)$ and 0.64 $(0.39,0.90)$, respectively. Additional file 15: Figure S13 showed the diagram of network meta-analysis between the changes of FEV1\% after using different statins in COPD patients. Network meta-analysis showed that Fluvastatin significantly increased compared with Atorvastatin, the SMD $(95 \% \mathrm{CI})$ was $0.79(0.03,1.41)$. The SUCRA of increasing the FEV1\% in COPD patients in Atorvastatin, Fluvastatin, Rosuvastatin, Pravastatin and Simvastatin were 17.0, 91.2, 11.0, 54.2 and $42.3 \%$,

Table 3 Rank probability analysis of PH with using stains in COPD patients

\begin{tabular}{llllll}
\hline Treatment & SUCRA & sd & $2.50 \%$ & median & $97.50 \%$ \\
\hline Atorvastatin & 75.4 & 0.2354 & 0.3 & 0.7 & 1.0 \\
Fluvastatin & 76.0 & 0.2616 & 0.3 & 0.7 & 1.0 \\
Simvastatin & 48.0 & 0.2299 & 0.3 & 0.3 & 1.0 \\
Conventional treatment & 0.6 & 0.0431 & 0.0 & 0.0 & 0.0 \\
\hline
\end{tabular}

respectively (Additional file 16: Table S3 and Additional file 17: Figure S14). Across all patients, Fluvastatin, Pravastatin and Simvastatin had higher overall probability, and they were more effective in increasing FEV1\% than other statins in COPD patients.

Additional file 18: Figure S15 showed that statins significantly increased FEV1/FVC\%, the SMD (95\% CI) was $0.35(0.23,0.47)$, with a significant strong heterogeneity $\left(I^{2}=81.3 \%\right)$. The heterogeneity significantly reduced when using sensitivity analysis and exclude one study [74] $\left(I^{2}=48.2 \%\right)$, the SMD $(95 \% \mathrm{CI})$ was $0.49(0.08$, 0.64). Subgroup analysis showed that Rosuvastatin, Simvastatin, Atorvastatin and Pravastatin were significantly increased FEV1/FVC\%, and the SMD $(95 \% \mathrm{CI})$ were $0.41(0.11,0.71), 0.53(0.34,0.72), 0.63(0.33,0.92)$ and $0.36(0.08,0.64)$, respectively. Additional file 19: Figure S16 showed the diagram of network meta-analysis between the changes of $\mathrm{FEV} 1 / \mathrm{FVC} \%$ after using different statins in COPD patients. Network meta-analysis showed, the SUCRA of increasing the FEV1/FVC\% in COPD patients in Atorvastatin, Fluvastatin, Rosuvastatin, Pravastatin and Simvastatin were 19.4, 93.3, 36.2, 45.3 and $30.3 \%$, respectively (Additional file 20: Table S4 and Additional file 21: Figure S17). Across all patients, Fluvastatin, Pravastatin and Rosuvastatin had higher overall probability, and they were more effective in increasing $\mathrm{FEV} 1 / \mathrm{FVC} \%$ than other statins in COPD patients.

Additional file 22: Figure S18 showed that statins significantly increased $6 \mathrm{MW}$, the SMD $(95 \% \mathrm{CI})$ was $0.54(0.38$, $0.71)$, with a significant lower heterogeneity $\left(I^{2}=40.8 \%\right)$. Subgroup analysis showed Atorvastatin and Simvastatin 


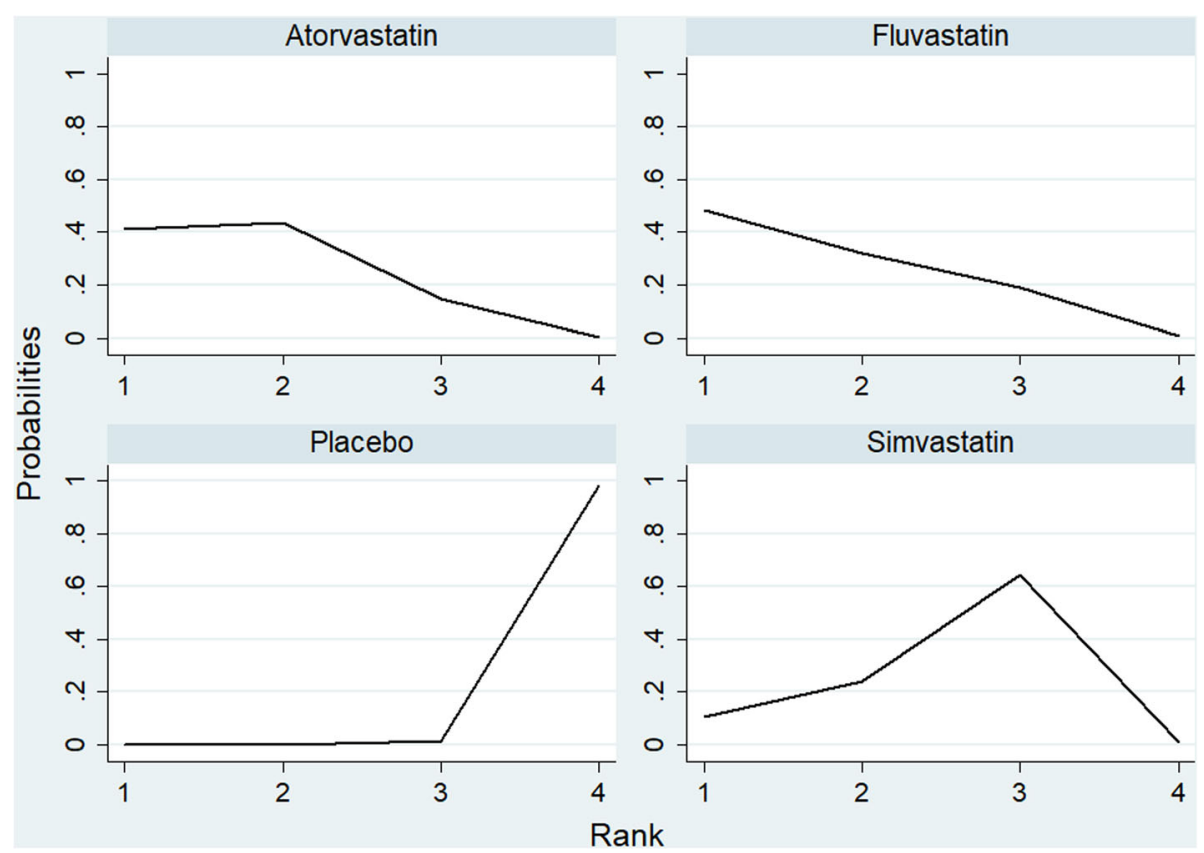

Fig. 7 Rank probability analysis of PH with using statins in COPD patients

were significantly increased 6WMD, and the SMD (95\% CI) were $0.42(0.14,0.70)$ and $0.79(0.52,1.05)$, respectively. Additional file 23: Figure S19 showed the diagram of network meta-analysis between the changes of $6 \mathrm{MW}$ after using different statins in COPD patients. Network meta-analysis showed, the SUCRA of increasing the 6 MW in COPD patients in Atorvastatin, Rosuvastatin, Pravastatin and Simvastatin were 30.5, 46.6, 43.1 and 47.8\%, respectively (Additional file 24: Table S5 and Additional file 25: Figure S20). Across all patients, Simvastatin and Rosuvastatin had higher overall probability, and they were more effective in increasing $6 \mathrm{MW}$ than other statins in COPD patients.

\section{Using statins reduced the level of TC and TG}

Additional file 26: Figure S21 showed that statins significantly reducing TC, the SMD $(95 \% \mathrm{CI})$ was $-0.86(-1.02$, $-0.70)$, with a significant moderate heterogeneity $\left(I^{2}=\right.$ $71.1 \%)$. The heterogeneity significantly reduced when using sensitivity analysis and exclude one study [74] $\left(I^{2}=\right.$ 53.7\%), the SMD (95\% CI) was - $0.75(-0.92,-0.57)$. Subgroup analysis showed that Pravastatin, Simvastatin, Atorvastatin an Fluvastatin significantly reduced $\mathrm{TC}$, and the SMD $(95 \%$ CI $)$ were - $1.02 \quad(-1.34,-0.70),-0.82 \quad(-$ $1.07,-0.57),-0.69(-1.30,-0.09)$ and $-1.58(-2.00,-1.15)$, respectively. Additional file 27: Figure S22 showed the diagram of network meta-analysis between the changes of TC after using different statins in COPD patients. Network meta-analysis showed that Fluvastatin significantly reduced TC compared Simvastatin, the SMD (95\% CI) was $-0.77(-1.47,-0.07)$. The SUCRA of reducing the TC in COPD patients in Atorvastatin, Fluvastatin, Rosuvastatin, Pravastatin and Simvastatin were 39.5, 96.9, 51.1, 65.1 and 46.4\%, respectively (Additional file 28: Table S6 and Additional file 29: Figure S23). Across all patients, Fluvastatin, Pravastatin and Rosuvastatin had higher overall probability, and they were more effective in reducing TC than other statins in COPD patients.

Additional file 30: Figure S24 showed that statins significantly reduced TG, the SMD $(95 \% \mathrm{CI})$ was -0.21 $(-0.39,-0.02)$, with a significant stronger heterogeneity $\left(I^{2}=87.7 \%\right)$. The heterogeneity significantly reduced when using sensitivity analysis and exclude one study [74] $\left(I^{2}=60.2 \%\right)$, the SMD $(95 \% \mathrm{CI})$ was -0.50 ($0.71,-0.29)$. Subgroup analysis showed Pravastatin and Atorvastatin were significantly reducing TG. The SMD $(95 \% \mathrm{CI})$ were $-0.66(-0.97,-0.35)$ and - $1.01(-1.55$, 0.47), respectively. Additional file 31: Figure S25 showed the diagram of network meta-analysis between the changes of TG using different statins in COPD patients. Network meta-analysis showed, Atorvastatin significantly reduced TG compared Fluvastatin, the SMD (95\% CI) was $-1.75(-3.13,-0.28)$. The SUCRA of reducing the TG in COPD patients in Atorvastatin, Fluvastatin, Rosuvastatin, Pravastatin and Simvastatin were 91.6, 5.3, 48.5, 78.7 and 39.4\%, respectively (Additional file 32: Table S7 and Additional file 33: Figure S26). Across all patients, Atorvastatin, Pravastatin and Rosuvastatin had higher overall probability, and they were more effective in reducing TG than other statins in COPD patients. 


\section{Sensitivity analysis and publication bias}

We saw heterogeneity among studies about the $I^{2}$ of all-cause mortality, heart disease-related mortality, COPD mortality and AECOPD were 86.8, 60.7, 95.7 and $78.4 \%$. The heterogeneity were significantly reduced after removing studies with a large heterogenous source through sensitivity analysis. The heterogeneity of inflammatory factors, lung function index, blood lipids index significantly reduced through removing studies with a large heterogenous source by sensitivity analysis or subgroups analysis. Begger regression tests provided no evidence of substantial publication bias $(P>0.05$ for all tests).

\section{Discussion}

Our study used general meta-analysis to estimate the relative risk of morality in using statins, and used network meta-analysis to estimate standardized mean difference between different statins, and to further determine their effectiveness in COPD in reducing inflammatory factors and increasing lung function index, and the most important is to find which statins have more effective for treating COPD patients. Our network meta-analysis not only included the long-term outcomes which involving all-cause and cause-specific mortality, but also included the recent outcomes which containing inflammatory factors, lung function index, blood lipids index and $\mathrm{PH}$, which comprehensively evaluated which statin is better for COPD patients and provide evidence for the clinical treatment.

The study found that using statins reduced $29 \%$ of the risk of all-cause mortality, 28\% COPD mortality and 16\% AECOPD, the RR (95\% CI) were $0.71(0.62,0.80), 0.72$ $(0.53,0.98)$ and $0.84(0.79,0.89)$, respectively. A recent meta-analysis found [16] using statins reduced all-cause mortality, COPD mortality and AECOPD which including twenty studies published 2017 years, the HR (95\% CI) were 0.65 (0.57-0.74), $0.41(0.28-0.59)$ and 0.58 (0.48-0.72), respectively. However, it didn't study COPD death. Zhang et al. found [17] that statins therapy was associated with reduced $\mathrm{pH}$ which including six studies, the SMD $(95 \% \mathrm{CI})$ was $-0.70(-0.99,-0.41)$. PH is a serious and progressive vascular disease, which is yet incurable and generally results in heart failure and death. Similarly, our study also found using statins reduced $\mathrm{PH}$ which including ten studies, the SMD (95\% CI) was 0.71 (- 0.85,-0.57). Subgroup analysis found that Simvastatin, Atorvastatin and Fluvastatin significantly reduced $\mathrm{PH}$, and the SMD $(95 \% \mathrm{CI})$ were $-0.54(-0.79,-0.30)$, $0.79(-0.99,-0.59)$ and $-0.77(-1.07,-0.46)$, respectively.

Network meta-analysis showed the SUCRA of Atorvastatin, Fluvastatin and Simvastatin were 75.4, 76.0 and 48.0\%, which means Atorvastatin and Fluvastatin are more effective than simvastatin. The well-known mechanisms for the occurrence and development of $\mathrm{PH}$ include pulmonary vascular remodeling, abnormal vasoconstriction, and thrombosis, which all lead to increased pulmonary arterial pressure and vascular resistance, as well as right heart failure. Seeming to aim at these pathophysiological alterations, statins have been shown to restrict vascular cell proliferation, improve endothelial function by increasing Nitric oxide (NO) production, and inhibiting thrombogenic response, thus may exert a potential therapeutic benefit [76, 77].

Chronic inflammation and oxidation reaction played a huge role in the progression of COPD [78]. The protective effect of statins could be explained by their pleiotropic effects, including inhibit vascular endothelial inflammatory response, stabilize athermanous plaque, antithrombotic effects, and improve endothelial function [79]. As COPD progresses, lots of inflammatory biomarkers, such as CRP, IL-6, IL-8, and TNF- $\alpha$ increased in COPD patients [80]. These numerous inflammatory responses are believed to contribute to disease progression of COPD, and systemic inflammation often together with COPD and elevated circulating inflammatory factors can be an important risk factor for mortality [81]. Our study found using statin reduced inflammatory mediators including CRP, IL-6, IL- 8 and TNF- $\alpha$, the SMD $(95 \% \mathrm{CI})$ were $-0.62(-0.52,-0.72),-0.95(-1.09,-0.81)$, $-0.37(-0.58,-0.16)$ and $-0.56(-0.72,-0.39)$, respectively. Subgroup analysis showed that there were different effectiveness using different statins in reducing inflammatory mediators. Network meta-analysis showed Fluvastatin (97.7\%), Atorvastatin and Rosuvastatin had higher overall probability, and they were more effective in reducing CRP than other statins in COPD patients; Simvastatin, Rosuvastatin and Pravastatin had higher overall probability, and they were more effective in reducing IL-6 than other statins in COPD patients. A large number of studies have showed the effect of statins to modulate the immune responses and reduce inflammatory mediators including CRP, IL-6, IL-8, and IL-17 in inflammatory based diseases [82, 83].

Wright et al. study showed that endothelin (ET) is a powerful pulmonary vasodilator and plays an important role in the pathogenesis and treatment of COPD patients [84]. NO has been known as maintain patency. The interaction between ET and NO together maintains the tone of the blood vessels. When vascular endothelial cells are damaged, endothelin is synthesized and released in large amounts, but it does not increase the synthesis and release of NO, resulting in an imbalance of ET and $\mathrm{NO}$, and the formation of pulmonary artery remodeling. Studies have shown that fluvastatin can increase the post-transcriptional activity of the eNOS gene, inhibition of endothelin synthesis and secretion, promotion of $\mathrm{NO}$ synthesis and secretion, and improve lung function [56, 
85]. Chronic inflammatory response can also stimulate vascular endothelial cells and smooth muscle cells to produce $\mathrm{ET}$, promote the formation of $\mathrm{PH}$. Atorvastatin can reduce the secretion of IL- 6 and TNF-a, reduce the release of CRP, reduce the inflammatory response, regulate the balance of $\mathrm{NO}$ and ET-1, and ultimately reduce the pulmonary artery pressure and Improve lung function by inhibiting the transcription of nuclear factor (NF)-KB [73, 82]. A common complication of COPD is $\mathrm{PH}$, which mechanism is complex and not fully understood. Study has found that chronic hypoxia induced $\mathrm{PH}$, the main mechanism may be associated with hypoxia-induced pulmonary vasoconstriction, which in turn leads to dysfunction of pulmonary vascular endothelial cells, promotes increased synthesis and clearance of ET-1, and inhibits NO synthesis and release, the pulmonary blood vessels gradually remodel and eventually develop into $\mathrm{PH}$ [86]. Soo speculates that CRP and ET-1 may be closely related to $\mathrm{PH}$ in patients with COPD [87]. Based on the results of this study, we speculated that statins can be used to prevent COPD progression and reduce $\mathrm{PH}$ by reducing CRP, IL- 6 and TNF- $a$ and other inflammatory factors.

The present study has a number of advantages over the previous meta-analysis. On the one hand, this study not only includes long-term outcome indicators such as the risk of AECOPD, heart disease-related mortality and all-cause mortality, but also includes inflammatory factors and lung function index, comprehensive analysis the effect of using statin in COPD patients. On the other hand, network meta-analysis studied which statin is better for treating COPD. Of course, there are some limitations of the study. Firstly, in the study of the risk of AECOPD, heart disease-related mortality and all-cause mortality, the original studies did not refer to specific statins, so only general meta-analysis was performed. Secondly, although our study included a comparison of the effects of different statins, the results which indirect were less effective than the direct comparisons. Thirdly, RCTs included in the study have a small sample size. Therefore, there is needed more and more randomized, well-designed, multi-center, direct comparison and double blind clinical studies to identify the long-term effects of statins in COPD patients.

In conclusion, our network meta-analysis showed that long-term using statins reduced inflammatory factors including CRP and IL-6, increased lung function index including $\mathrm{FEV} 1 \%$ and $\mathrm{FEV} 1 / \mathrm{FVC} \%$, and reduced the risk of AECOPD, heart disease-related mortality and all-cause mortality. In addition, Fluvastatin, Atorvastatin and Rosuvastatin are more effective in COPD patients considered comprehensively.

\section{Conclusion}

Using statins can reduce the risk of mortality, the level of CRP and PH in COPD patients. In addition, Fluvastatin and Atorvastatin are more effective in reducing CRP and $\mathrm{PH}$ in COPD patients.

\section{Additional files}

Additional file 1: Flow chart of studies search and studies selection. (TIF $12473 \mathrm{~kb}$ )

Additional file 2: Forest plot showing effect of statins on heart diseaserelated mortality in COPD patients. (TIF $39534 \mathrm{~kb}$ )

Additional file 3: Forest plot showing effect of statins on COPD mortality in COPD patients. (TIF 39315 kb)

Additional file 4: Forest plot showing effect of statins on AECOPD in COPD patients. (TIF $36283 \mathrm{~kb}$ )

Additional file 5: Forest plot showing effect of statins on IL-6 in COPD patients. (TIF $29566 \mathrm{~kb}$ )

Additional file 6: Evidence network of the effect of using statins on IL-6 levels in COPD patients. (TIF $14099 \mathrm{~kb}$ )

Additional file 7: Rank probability analysis of IL-6 with using statins in COPD patients. (PDF $177 \mathrm{~kb}$ )

Additional file 8: Rank probability analysis of IL-6 with using statins in COPD patients. (TIF 36014 kb)

Additional file 9: Forest plot showing effect of statins on IL-8 in COPD patients. (TIF $34035 \mathrm{~kb}$ )

Additional file 10: Forest plot showing effect of statins on TNF-a in COPD patients. (TIF $34092 \mathrm{~kb}$ )

Additional file 11: Evidence network of the effect of using statins on TNF-a levels in COPD patients. (TIF 8093 kb)

Additional file 12: Rank probability analysis of TNF-a with using statins in COPD patients. (PDF $272 \mathrm{~kb}$ )

Additional file 13: Rank probability analysis of TNF-a with using statins in COPD patients. (TIF $36012 \mathrm{~kb}$ )

Additional file 14: Forest plot showing effect of statins on FEV1\% in COPD patients. (TIF $28073 \mathrm{~kb}$ )

Additional file 15: Evidence network of the effect of using statins on FEV1\% in COPD patients. (TIF $13811 \mathrm{~kb}$ )

Additional file 16: Rank probability analysis of FEV1\% with using statins in COPD patients. (PDF $177 \mathrm{~kb}$ )

Additional file 17: Rank probability analysis of FEV1\% with using statins in COPD patients. (TIF $36014 \mathrm{~kb}$ )

Additional file 18: Forest plot showing effect of statins on FEV1/FVC\% in COPD patients. (TIF $28661 \mathrm{~kb}$ )

Additional file 19: Evidence network of the effect of using statins on FEV1/FVC\% in COPD patients. (TIF $13672 \mathrm{~kb}$ )

Additional file 20: Rank probability analysis of FEV1/FVC\% with using statins in COPD patients. (PDF $177 \mathrm{~kb}$ )

Additional file 21: Rank probability analysis of FEV1/FVC\% with using statins in COPD patients. (TIF 36014 kb)

Additional file 22: Forest plot showing effect of statins on 6MW in COPD patients. (TIF $32244 \mathrm{~kb}$ )

Additional file 23: Evidence network of the effect of using statins on 6MW in COPD patients. (TIF $13184 \mathrm{~kb}$ )

Additional file 24: Rank probability analysis of 6MW with using statins in COPD patients. (PDF $176 \mathrm{~kb}$ )

Additional file 25: Rank probability analysis of 6MW with using statins in COPD patients. (TIF $36013 \mathrm{~kb}$ )

Additional file 26: Forest plot showing effect of statins on TC in COPD patients. (TIF 31508 kb) 
Additional file 27: Evidence network of the effect of using statins on TC levels in COPD patients. (TIF $13642 \mathrm{~kb}$ )

Additional file 28: Rank probability analysis of TC with using statins in COPD patients. (PDF $177 \mathrm{~kb}$ )

Additional file 29: Rank probability analysis of TC with using statins in COPD patients. (TIF 36014 kb)

Additional file 30: Forest plot showing effect of statins on TG in COPD patients. (TIF $32559 \mathrm{~kb}$ )

Additional file 31: Evidence network of the effect of using statins on TG levels in COPD patients. (TIF 13633 kb)

Additional file 32: Rank probability analysis of TG with using statins in COPD patients. (PDF $177 \mathrm{~kb}$ )

Additional file 33: Rank probability analysis of TG with using statins in COPD patients. (TIF $36014 \mathrm{~kb}$ )

\section{Acknowledgments}

Not applicable.

\section{Funding}

The authors received no specific funding for this work.

\section{Availability of data and materials}

Authors are available to include the dataset supporting the conclusions of this article as "Additional file".

\section{Author's contributions}

$Y L, R C$ contributed to the study concept and design. RC, XX and JY performed the study search and extracted data. $Y L$ and $Y T$ did analysis and interpretation of data. YL wrote the first draft, RC and NC critically revised the manuscript for important intellectual content.All authors read and approved the final manuscript.

\section{Ethics approval and consent to participate}

Not applicable.

\section{Consent for publication}

Not applicable.

\section{Competing interests}

The authors declare that they have no competing interests.

\section{Publisher's Note}

Springer Nature remains neutral with regard to jurisdictional claims in published maps and institutional affiliations.

\section{Author details}

'The First Hospital of Lanzhou University, Lanzhou, Gansu, People's Republic of China. 'Lanzhou Maternal and Child Health Care Hospital, Lanzhou, Gansu 730000, People's Republic of China. ${ }^{3}$ Lanzhou University, Basic Medical College, Lanzhou, Gansu 730000, People's Republic of China.

Received: 31 July 2018 Accepted: 13 January 2019

Published online: 23 January 2019

\section{References}

1. Marin L, Colombo P, Bebawy M, Young PM, Traini D. Chronic obstructive pulmonary disease: patho-physiology, current methods of treatment and the potential for simvastatin in disease management. Expert Opinion on Drug Delivery. 2011;8:1205.

2. Adeloye D, Chua S, Chinwei L, Basquill C, Papana A, Theodoratou E, Nair H, Gasevic D, Sridhar D, Campbell H. Global and regional estimates of COPD prevalence: systematic review and meta-analysis. J Glob Health. 2015;5: 020415

3. Chen W, Thomas J, Sadatsafavi M, Fitzgerald JM. Risk of cardiovascular comorbidity in patients with chronic obstructive pulmonary disease: a systematic review and meta-analysis. Lancet Respiratory Medicine. 2015;3: 631-9.
4. Curkendall SM, Deluise C, Jones JK, Lanes S, Stang MR, Jr GE, She D. Cardiovascular disease in patients with chronic obstructive pulmonary disease, Saskatchewan Canada cardiovascular disease in COPD patients. Annals of Epidemiology. 2006;16:63.

5. Javad MSA, Hanieh R, Masoomeh F, Rostam Y, Mansour E. Evaluation of the effects of atorvastatin on the treatment of secondary pulmonary hypertension due to chronic obstructive pulmonary diseases: a randomized controlled trial. Iran Red Crescent Med J. 2013;15:649-54.

6. Lee JH, Lee DS, Kim EK, Choe KH, Oh YM, Shim TS, Kim SE, Lee YS, Lee SD. Simvastatin inhibits cigarette smoking-induced emphysema and pulmonary hypertension in rat lungs. Respiratory Medicine Copd Update. 2005;1:61-2.

7. Sin DD, Anthonisen NR, Soriano JB, Agusti AG. Mortality in COPD: role of comorbidities. Eur Respir J. 2006:28:1245-57.

8. Fabbri LM, Rabe KF. From COPD to chronic systemic inflammatory syndrome? Lancet. 2007:370:797-9.

9. Gan WQ, Man SF, Senthilselvan A, Sin DD. Association between chronic obstructive pulmonary disease and systemic inflammation: a systematic review and a meta-analysis. Thorax. 2004;59:574-80.

10. Agustí A, Faner R. Systemic inflammation and comorbidities in chronic obstructive pulmonary disease. Proc Am Thorac Soc. 2012;9:43-6.

11. Lahousse L, Loth DW, Joos GF, Hofman A, Leufkens HG, Brusselle GG, Stricker BH. Statins, systemic inflammation and risk of death in COPD: the Rotterdam study. Pulm Pharmacol Ther. 2013;26:212-7.

12. Lu Y, Cheng Z, Zhao Y, Chang X, Chan C, Bai Y, Cheng N. Efficacy and safety of long-term treatment with statins for coronary heart disease: a Bayesian network meta-analysis. Atherosclerosis. 2016;254:215-27.

13. Desai CS, Martin SS, Blumenthal RS. Non-cardiovascular effects associated with statins. BMJ. 2014;349:93743.

14. Young RP, Hopkins RJ. The mevalonate pathway and innate immune hyperresponsiveness in the pathogenesis of COPD and lung cancer: potential for chemoprevention. Curr Mol Pharmacol. 2017;10:15-21.

15. Cao C, Wu Y, Xu Z, Lv D, Zhang C, Lai T, Li W, Shen H. The effect of statins on chronic obstructive pulmonary disease exacerbation and mortality: a systematic review and meta-analysis of observational research. Sci Rep. 2015;5:16461.

16. Li W, Huang $Y$, Cheng $H$, Feng $Y$. Statins reduce all-cause mortality in chronic obstructive pulmonary disease: an updated systematic review and meta-analysis of observational studies. Oncotarget. 2017:8:73000-8.

17. Zhang MZ, Qian DH, Xu JC, Yao W, Fan Y, Wang CZ. Statins may be beneficial for patients with pulmonary hypertension secondary to lung diseases. Journal of Thoracic Disease. 2017;9:2437.

18. Zhou Y, Wang J, Zhang L. Statin therapy on pulmonary function in patients with COPD: a meta-analysis of randomized controlled trials. Chinese pharmacy. 2018;4(27):281-8.

19. Wells GA, Shea BJ, O'Connell D, Peterson J, Welch V, Losos M, Tugwell P. The Newcastle-Ottawa Scale (NOS) for Assessing the Quality of NonRandomized Studies in Meta-Analysis. Applied Engineering in Agriculture. 2014;18:727-34

20. Higgins JP, Altman DG, Gøtzsche PC, Jüni P, Moher D, Oxman AD, Savovic J, Schulz KF, Weeks L, Sterne JA. The Cochrane Collaboration's tool for assessing risk of bias in randomised trials. Bmj British Medical Journal. 2011;343:889-93.

21. Dersimonian R, Nan L. Meta-analysis in clinical trials revisited. Contemporary Clinical Trials. 2015:45:139-45.

22. Higgins JPT, Thompson SG. Quantifying heterogeneity in meta-analysis. Stat Med. 2002:21:1539-58

23. LU G, Ades AE. Combination of direct and indirect evidence in mixed treatment comparisons. Stat Med. 2004;23:3105-24.

24. Salanti G, Ades AE, loannidis JP. Graphical methods and numerical summaries for presenting results from multiple-treatment meta-analysis: an overview and tutorial. J Clin Epidemiol. 2011;64:163-71.

25. Bartziokas K, Papaioannou Al, Minas M, Kostikas K, Banya W, Daniil ZD, Haniotou A, Gourgoulianis Kl. Statins and outcome after hospitalization for COPD exacerbation: a prospective study. Pulm Pharmacol Ther. 2011;24: 625-31.

26. Rossi A, Inciardi RM, Rossi A, Temporelli PL, Lucci D, Gonzini L, Marchioli R, Nicolosi GL, Tavazzi L. Prognostic effects of rosuvastatin in patients with coexisting chronic obstructive pulmonary disease and chronic heart failure: a sub-analysis of GISSI-HF trial. Pulm Pharmacol Ther. 2017:44:16-23.

27. van Gestel YR, Hoeks SE, Sin DD, Hüzeir V, Stam H, Mertens FW, van Domburg RT, Bax JJ, Poldermans D. COPD and cancer mortality: the influence of statins. Thorax. 2009;64:963. 
28. Dangestel YRBM, Hoeks SE, Sin DD. Effect of Statin Therapy on Mortality in Patients with Peripheral Arterial Disease and Comparison of those With versus Without Associated Chronic Obstructive Pulmonary Disease. Am J Cardiol. 2008:48:1062.

29. Mortensen EM, Copeland LA, Pugh MJV, Restrepo MI, Molina RMD, Nakashima B, Anzueto A. Impact of statins and ACE inhibitors on mortality after COPD exacerbations. Respir Res. 2009;10:45.

30. Criner GJ, Connett JE, Aaron SD, Albert RK, Bailey WC, Casaburi R, Cooper JA Jr, Curtis JL, Dransfield MT, Han MK, et al. Simvastatin for the prevention of exacerbations in moderate-to-severe COPD. N Engl J Med. 2014;370:2201-10.

31. Lawes CM, Thornley S, Young R, Hopkins R, Marshall R, Chan WC, Jackson G. Statin use in COPD patients is associated with a reduction in mortality: a national cohort study. Prim Care Respir J. 2012;21:35.

32. Ekstrom MP, Hermansson AB, Strom KE, editors. Effect Of Cardiovascular Drugs On Mortality In Severe Chronic Obstructive Pulmonary Disease: A Time-Dependent Analysis. San Francisco: American Thoracic Society 2012 International Conference; 2012. May 18-23, 2012

33. Søyseth V, Brekke PH, Smith P, Omland T. Statin use is associated with reduced mortality in COPD. Eur Respir J. 2007;29:279-83.

34. Raymakers AJ, Sadatsafavi M, Sin DD, De Vera MA, Lynd LD. The impact of statin use on all-cause mortality in patients with COPD: a population based cohort study. Chest. 2017;152(3):486-93.

35. Frost FJ, Petersen H, Tollestrup K, Skipper B. Influenza and COPD mortality protection as pleiotropic, dose-dependent effects of statins. Chest. 2007;131: $1006-12$.

36. Sheng X, Murphy MJ, Macdonald TM, Schembri S, Simpson W, Winter J, Winter $J H$, Wei L. Effect of statins on total cholesterol concentrations, cardiovascular morbidity, and all-cause mortality in chronic obstructive pulmonary disease: a population-based cohort study. Clin Ther. 2012;34:374-84.

37. Su TH, Chang SH, Chen PC, Chan YL. Temporal trends in treatment and outcomes of acute myocardial infarction in patients with chronic obstructive pulmonary disease: a Nationwide population-based observational study. J Am Heart Assoc. 2017;6.

38. Dong $\mathrm{P}$, Jin $\mathrm{H}$, Zengfan L. Comparative study of atorvastatin and simvastatin in the treatment of chronic obstructive pulmnary disease. China modern medicine. 2015;22:132-4.

39. Liu JC, Yang TY, Hsu YP, Hao WR, Kao PF, Sung LC, Chen CC, Wu SY. Statins dose-dependently exert a chemopreventive effect against lung cancer in COPD patients: a population-based cohort study. Oncotarget. 2016;7:59618-29.

40. Huang CC, Chan WL, Chen YC, Chen TJ, Chou KT, Lin SJ, Chen JW, Leu HB. Statin use and hospitalization in patients with chronic obstructive pulmonary disease: a nationwide population-based cohort study in Taiwan. Clin Ther. 2011;33:1365-70.

41. Ingebrigtsen TS, Marott JL, Nordestgaard BG, Lange P, Hallas J, Vestbo J. Statin use and exacerbations in individuals with chronic obstructive pulmonary disease. Thorax. 2015;70:33-40.

42. Ajmera M, Shen C, Sambamoorthi U. Association between statin medications and COPD-specific outcomes: a real-world observational study. Drugs - Real World Outcomes. 2017:4:9-19.

43. Wang MT, Lo MW, Tsai CL, Chang LC, Malone DC, Chu CL, Liou JT. Statin use and risk of COPD exacerbation requiring hospitalization. Am J Med. 2013;126:598.

44. Mao L, Ying L, Hua Q. Effects of atorvastatin on serum levels of inflammatoryfactors, pulmonary function and right ventricular remodeling in patients with chronic obstructive pulmonary disease compicated with pulmonary hypertensin. Drug evaluation research. 2017;40(9):1323-6.

45. Yan D. Clinical efficacy of simvastatin in treatment of COPD complicated with pulmonary hypertenson. Pharmacy today. 2016;26(8):606-8.

46. Wu Y, Liao L. Clinical effects of simvastatin on patients with COPD and its effects on C-reactive protein, prealbumin, and pulmonary function. Hainan Med J. 2016;27(23):3808-10.

47. Lee TM, Lin MS, Chang NC. Usefulness of C-reactive protein and interleukin6 as predictors of outcomes in patients with chronic obstructive pulmonary disease receiving pravastatin. Am J Cardiol. 2008;101:530-5.

48. Undas A, Kaczmarek P, Sladek K, Stepien E, Skucha W, Rzeszutko M, Gorkiewiczkot I, Tracz W. Fibrin clot properties are altered in patients with chronic obstructive pulmonary disease. Beneficial effects of simvastatin treatment. Thromb Haemost. 2009;102:1176-82.

49. Ghobadi H, Lari SM, Pourfarzi F, Mahmoudpour A, Ghanei M. The effects of atorvastatin on mustard-gas-exposed patients with chronic obstructive pulmonary disease: a randomized controlled trial. J Res Med Sci. 2014;19:99-105.
50. Neukamm A, Høiseth AD, Einvik G, Lehmann S, Hagve TA, Søyseth V, Omland T. Rosuvastatin treatment in stable chronic obstructive pulmonary disease (RODEO): a randomized controlled trial. J Intern Med. 2015;278:59-67.

51. Kaczmarek P, Sładek K, Skucha W, Rzeszutko M, Iwaniec T, Dziedzina S, Szczeklik A. The influence of simvastatin on selected inflammatory markers in patients with chronic obstructive pulmonary disease. Pol Arch Med Wewn. 2010;120:11-7.

52. Fengxia Y, Haixia Y, Mingzhong F, Lijun K, Changhua Q, Shujuan W, Yuguang J, Lingting K, Fei L. Effects of atorvastatin on pulmonary hypertension in chronic obstructive pulmonary disease. Chin J Clinicians. 2012;6(5):1129-33.

53. Guo S, Lina N, Lixin N, Shengxia X. Effect of atorvastatin calcium on serum IL-13, TNF- $a$ and CRP in patients with chronic obstructive pulmonary disease. Clin Pulm Med. 2015;20(5):883-5.

54. Rongchang Z, Shuai Z, Luo Y. The effect of atorvastatin calcium ablets on Creactive protein, interleukin- 6 and pulmonary function in chronic obstructive pulmonary disease patients of stationary phase. Translational Medicine Journal. 2015;4(5):282-4.

55. Lina N, Guo S, Lixin N, Shengxia X. Clinical observation of atorvastatin in treatment of chronic obstructive pulmonary disease with pulmonary hypertension. Clin Pulm Med. 2015;20(11):2114-6.

56. Wang $L$, Mengjue $L$, Yanping T. Therapeutic effect of fluvastatin on pulmonary hypertension in patients with chronic obstructive pulmonary disease. Chin J Gerontol. 2011;31(9):1666-7.

57. Dong W, Ping K, Xiangzhu Z, Lin T, Cheng J, Cheng H, Weifeng P. Efficacy of statin in treatment of patients with chronic obstructive pulmonary disease at stable stage. Practical Journal of Medicine. 2015;31(11):1835-7.

58. Xuelin M, Wang Y. The influence of simvastatin with stable COPD patient. Practical Journal of Cardiovascular and Pulmonary Disease. 2011;19(44):554-6.

59. Wei Z, JinYong L, Weiyang F, Zhang Y. Clinical efficac of simvastatin and exercise training therapy in the treatment of chronic obstructive pulmonary disease patients complicated with metabolic syndrome and observation of its related indicators. Clin Pulm Med. 2016;21(1):81-3.

60. Chen T. Effect of simvastatin treatment on cardiopulmonary function and inflammatory cytokines in patients with COPD complicated with $\mathrm{PH}$. Modern Journal of Intergated Traditional Chinese and Western Medicine. 2017;26(8):851-3.

61. Ruihua Z, Lin Q, Wang X. Effect of simvastatin on cytokine levels and pulmonary function in elderly patients with COPD complicated with $\mathrm{PH}$. Chin J Gerontol. 2015;12(35):7105-7.

62. Hong L, Zhengzheng T, Guiqin F, Lu B. Effects of simvastatin on patients with chronic obstructive pulmonary disease. Modern Medical Journal. 2017; 45(3):575-7.

63. Juan S, Yipeng D. Curative effect of simvastatin in the treatment of chronic obstructive pulmonary disease complicated with pulmonary arterial hypertension. Hainan Med J. 2014;25(2):164-6.

64. Qing Y, Kuang J. Effect of low dose simvastatin in patients with chronic obstructive pulmonary disease complicated with pulmonary arterial hypertension. J Med Research. 2015;44(1):107-9.

65. Zheng W, Wu J. Effect of atorvastatin on inflammatory factors, pulmonary function and quality of life of patients with chronic obstructive pulmonary diseases. Clin Pulm Med. 2013;18(5):870-3.

66. Hang $M, H e H_{1}$ Haiyan Q, Yijiang T. Effects of atorvastatin on patients with chronic obstructive pulmonary disease combined with pulmonary hypertension. J Shanxi Med Univ. 2014;45(3):204-6.

67. Xu J, Zheng G, Shengjun $Y, W u$ J. The effect of fluvastatin on pulmonary function and interleukin- 6 levels in patients with chronic obstructive pulmonary disease in stationary phase. China Medical Herald. 2012;9(25):98-101.

68. Zhimin J, Fengyuan L, Xufang T, Yang M, Liang Z, Changlu K. Effect of simvastatin on pulmonary function in patients with chronic obstructive pulmonary disease in stationary phase and study on mechanism. Hainan Med J. 2013;24(9):1277-9.

69. Yong Y, Minhua Z, Qiang T. The influence and curative effect of atorvastatin on plasma level of inflammatory cytokines of patients with chronic obstructive pulmonary disease. Chin J Pharmacoepidemiol. 2013;22(2):57-9.

70. Zhenghua $\mathrm{G}$, Jianan $\mathrm{H}$. The observation of rosuvastatin affect on IL-8 and the quality of life on COPD patients. Military Medical Journal of Southeast China. 2015;17(1):57-9. 
71. Lee TM, Chen CC, Shen HN, Chang NC. Effects of pravastatin on functional capacity in patients with chronic obstructive pulmonary disease and pulmonary hypertension. Clin Sci. 2009;116:497.

72. Shanshan C, Yong B, Furui Z, Guojun Z. Influence of atorvastatin on inflammatory markers in patients with chronic obstructive pulmonary disease and coronary heart disease. Journal of Clinical Medicine in Practice. 2015;19(17):5-7.

73. Dan C, Wang B. The clinical effect of atorvastatin in the treatment of chronic obstructive pulmonary disease complicated with pulmonary hypertension. Chin J of Clinical Rational Drug Use. 2016;9(2):1-3.

74. Wang W, Lin J. Fluvastatin in the treatment of 56 patients with chronic obstructive pulmonary disease complicated with pulmonary hypertension. Medical herald. 2012;31(9):1163-5.

75. Zheng G, Rongxing F, Liang D, Junxing F, Deng J. The effect of simvastatin treatment of chronic obstructive pulmonary disease (COPD) and its influence on patients with BNP paribas. China Modern medicine. 2017; 24(16):45-8.

76. Athyros VG, Kakafika Al, Tziomalos K, Karagiannis A, Mikhailidis DP. Pleiotropic effects of statins--clinical evidence. Curr Pharm Des. 2009;15: 479-89.

77. Katsiki N, Tziomalos K, Chatzizisis Y, Elisaf M, Hatzitolios Al. Effect of HMGCoA reductase inhibitors on vascular cell apoptosis: beneficial or detrimental? Atherosclerosis. 2010;211:9-14.

78. Pauwels RA, Buist AS, Calverley PM, Jenkins CR, Hurd SS. Global strategy for the diagnosis, management, and prevention of chronic obstructive pulmonary disease. NHLBI/WHO global initiative for chronic obstructive lung disease (GOLD) workshop summary. Am J Respir Crit Care Med. 2001; 176:1256-76.

79. Young RP, Hopkins R, Eaton TE. Potential benefits of statins on morbidity and mortality in chronic obstructive pulmonary disease: a review of the evidence. Postgrad Med J. 2009;85:414-21.

80. Howard ML, Vincent AH. Statin effects on exacerbation rates, mortality, and inflammatory markers in patients with chronic obstructive pulmonary disease: a review of prospective studies. Pharmacotherapy. 2016;36:536-47.

81. John ME, Cockcroft JR, Mckeever TM, Coward WR, Shale DJ, Johnson SR, Thornton JG, Harrison TW, Knox AJ, Bolton CE. Cardiovascular and inflammatory effects of simvastatin therapy in patients with COPD: a randomized controlled trial. International Journal of Chronic Obstructive Pulmonary Disease. 2015;10:211-21.

82. Bonnet J, Mcpherson R, Tedgui A, Simoneau D, Nozza A, Martineau P, Davignon J, Investigators C. Comparative effects of 10-mg versus 80-mg atorvastatin on high-sensitivity C-reactive protein in patients with stable coronary artery disease: results of the CAP (comparative atorvastatin pleiotropic effects) study. Clin Ther. 2008;30:2298-313.

83. Pruefer D, Makowski J, Schnell M, Buerke U, Dahm M, Oelert H, Sibelius U, Grandel U, Grimminger F, Seeger W. Simvastatin inhibits inflammatory properties of Staphylococcus aureus alpha-toxin. Circulation. 2002;106:2104.

84. Wright JL, Levy RD, Churg A. Pulmonary hypertension in chronic obstructive pulmonary disease: current theories of pathogenesis and their implications for treatment. Thorax. 2005:60:605.

85. Murata T, Kinoshita K, Hori M, Kuwahara M, Tsubone H, Karaki H, Ozaki H. Statin protects endothelial nitric oxide synthase activity in hypoxia-induced pulmonary hypertension. Arteriosclerosis Thrombosis \& Vascular Biology. 2005;25:2335.

86. Khmel'Kova MA, Shmelev El. Pulmonary hypertension in chronic obstructive lung disease. Problemy Tuberkuleza I Bolezneĭ Legkikh. 2004;90(11):3-12.

87. Soo KY, Young CS, Joon SH, Young KE, Kab YB, Jung BH, Jae Ol, Sik KK, Chul KY, Chul LS. Plasma C-reactive protein and Endothelin-1 level in patients with chronic obstructive pulmonary disease and pulmonary hypertension. J Korean Med Sci. 2010;25(10):1487-91.

Ready to submit your research? Choose BMC and benefit from:

- fast, convenient online submission

- thorough peer review by experienced researchers in your field

- rapid publication on acceptance

- support for research data, including large and complex data types

- gold Open Access which fosters wider collaboration and increased citations

- maximum visibility for your research: over $100 \mathrm{M}$ website views per year

At BMC, research is always in progress.

Learn more biomedcentral.com/submissions 